Ann. Biol. anim. Bioch. Biophys., 1979, 19 (1 B), 151-158.

\title{
Evaluation du comportement alimentaire maternel. Ses conséquences sur le poids fœtal
}

par R. FRYDMAN, J. C. BERARDI, Michèle BACHELIER, A. SPIRA, E. PAPIERNIK

\author{
Service de Gynécologie-Obstétrique, Hôpital Antoine Béclère, \\ Faculté de Médecine Paris Sud, 157, rue de la Porte de Trivaux, \\ 92141 Clamart, France.
}

Summary. An evaluation of maternal eating behavior and its effects on fetal weight.

Newborn mortality and disease are directly related to birthweight, and insufficient or excess weight at birth is always accompanied by an increase of these factors. In this report we attempted to determine if there was any relation between the nutritional status of the mother and the birthweight of the infant by evaluating the dief spontaneously eaten by women before pregnancy, at mid-pregnancy and during the last trimester. The preliminary results of this study indicated the significance of nutritional status before pregnancy and that the diet during the last trimester was especially important since it had considerable effect on the phase of rapid growth in the newborn.

\section{Introduction.}

Un regain d'intérêt concernant le comportement alimentaire maternel au cours de la gestation s'est fait jour depuis une décennie. Les effets de la sous-alimentation maternelle sur le fœtus sont bien connus dans le règne animal : réduction du poids de naissance, plus grande fragilité vis-à-vis des diverses agressions. Dans l'espèce humaine, le pourcentage des enfants de petit poids est significativement plus élevé chez les mères au faible gain pondéral gestationnel (Bergner et Susser, 1970, Eastman et Jackson, 1968). Les études pratiquées dans les pays en voie de développement (Lechtig et al., 1975) ou en pays développé lors des périodes de restrictions alimentaires, montrent qu'un déficit calorique maternel entraîne une diminution du poids du nouveau-né (Hytten et Leitch, 1971). Les nouveau-nés de faible poids de naissance pour leur âge gestationnel ont une mortalité périnatale supérieure à celle des nouveau-nés de poids normal. Leur avenir lointain apparaît grêvé d'un risque non négligeable de séquelles neurologiques et intellectuelles (Fitzhardinge et Steven, 1972; Thomson et Hytten, 1973 ; Singer et al., 1968).

A l'opposé, lorsque s'associent obésité et diabète maternel, la macrosomie fœtale est fréquente, l'obésité demeurant le fait déterminant. La mortalité périnatale des gros enfants est de 3 à 7 fois supérieure à celle des nouveau-nés de poids normal. 11 p. 100 présentent des troubles neurologiques ultérieurs (Sack, 1969). Donc, l'avenir des 
nouveau-nés est en partie fonction de leur poids de naissance (Wynn et Wynn, 1975 ; Naeye et al., 1973). Les femmes gestantes représentent un large éventail de comportements alimentaires. Beaucoup sont suralimentées, comme l'est le reste de la population française, mais une proportion non négligeable d'entre elles réduisent spontanément leur régime de crainte de prendre trop de poids. A l'inverse, une ration calorique très élevée peut être observée chez certaines femmes obèses.

La présente étude vise à décrire le comportement alimentaire spontané maternel au cours du troisième trimestre de la gestation ef de le corréler avec l'état de santé du nouveau-né afin d'établir les bases objectives du dépistage d'une population maternelle « à risque » d'insuffisance ou d'excès de croissance fœtale, les étiologies autres que nutritionnelles étant par ailleurs recherchées.

\section{Matériel et méthodes.}

A la maternité Antoine Béclère, au début du troisième trimestre de la grossesse (entre la $28^{\mathrm{e}}$ et la $32^{\mathrm{e}}$ semaine d'aménorrhée), une évaluation tant quantitative que qualitative de la ration calorique maternelle associée à une étude biochimique et hormonale de la glyco-régulation est pratiquée pour trois groupes de gestantes : les grossesses à risque d'enfants hypotrophiques constituent le groupe l, les grossesses normales constituent le groupe 11 et les grossesses à risque de macrosomie foetale constituent le groupe III.

1. - Pour le groupe I (grossesses à risque d'hypotrophie fœtale - $\mathrm{P}_{\mathrm{I}}$ ).

Entrent dans ce groupe les femmes présentant :

- Antécédent d'enfant hypotrophique ;

- Stature maternelle petite : index $\frac{P}{T}$ calculé en fonction de la table de référence (Metropolitan Life) ;

- Hauteur uférine inférieure au $10^{\mathrm{e}}$ percentile de la courbe de référence (Leroy et Lefort, 1971) ;

- Périmètre ombilical inférieur au 10 e percentile de la courbe de référence (Leroy et Lefort, 1971) ;

- Prise de poids inférieure à $3 \mathrm{~kg}$ à 30 semaines d'aménorrhée ;

- Mesure du diamètre bi-pariétal el surtout rapport bi-pariétal/bi-abdominal à l'échographie, par rapport aux courbes de référence du service.

Dans ce groupe, les autres étiologies du retard de croissance intra-utérin seront notées : hypertension, malformation utérine, tabagisme, malformation fœtale et fœtopathie.

2. - Pour le groupe $/ /\left(\mathrm{P}_{\mathrm{II}}\right)$.

Il s'agit de grossesses témoins, choisies parmi les grossesses sans pathologie, et acceptant une participation volontaire à cette enquête. 
3. - Pour le groupe III (risque de macrosomie fotale - $\mathrm{P}_{\mathrm{III}}$ ).

- Femme ayant un antécédent de gros enfant ;

- Femme obèse, en fonction de la table de référence (Metropolitan Life) ;

- Antécédents familiaux de diabète, de gros enfants ou d'obésité ;

- Gain pondéral supérieur à $10 \mathrm{~kg}$ à 30 semaines d'aménorrhée.

A la naissance, l'âye gestationnel, le poids, la taille, le sexe, les périmètres crânien et thoracique ainsi que le poids du placenta sont notés.

L'enquête diététique. - L'évaluation de la ration calorique ingérée a été réalisée à l'aide d'un semainier adressé par la diététicienne à chaque patiente à la $30 \mathrm{e}, 34^{\mathrm{e}}$ et $38^{\mathrm{e}}$ semaine de grossesse, après qu'une première consultation de diététique (à 30 semaines) ait évalué par l'interrogatoire la ration spontanée des 24 h précédentes (Pequinot et Cubeau, 1973). Sur le semainier est retranscrite la pesée des aliments quotidiennement ingérés au cours de la semaine étudiée. Chaque repas est ainsi analysé en détail, permettant de calculer le rapport en protides, lipides, glucides, alcool (s'il y a lieu). En ce qui concerne les glucides, la proportion d'amidon ef de saccharose est notée.

La diététicienne procède à une évaluation de l'histoire diététique de la patiente et tente de caractériser les déterminants du comportement alimentaire (contraintes économiques et professionnelles, habitudes nutritionnelles, familiales, craintes de la difformité, rejet inconscient de la grossesse). Pour toutes les femmes, la consommation de tabac est précisée. Les différents semainiers permettent de vérifier la stabilité ou la variation de la ration calorique.

\section{Résultats et discussion.}

Le tableau 1 indique le poids et la taille des nouveau-nés. On constate que les groupes $P_{I}$ et $P_{I I}$, c'est-à-dire les mères parmi lesquelles une prévision de petits ou de gros enfants avait été faite, se subdivisent en deux sous-groupes: un groupe $P_{I} N_{I}$ où, effectivement, les enfants sont petits (inférieur au $10^{\mathrm{e}}$ percentile de la courbe LeroyLefort) et un groupe $P_{I} N_{I I}$ où les enfants présentent un poids normal. La situation est similaire pour le groupe $P_{I I I}$. Parmi les enfants normaux prévus (groupe $P_{I I}$ ), seuls

\section{TABLEAU 1}

Poids ef taille des nouveau-nés issus des 3 groupes mafernels

\begin{tabular}{|c|c|c|c|c|c|}
\hline & \multicolumn{5}{|c|}{ Groupes mafernels } \\
\hline & \multicolumn{2}{|c|}{$P_{\mathbf{I}}$} & \multirow{2}{*}{$\frac{P_{I I}}{N_{I I}}$} & \multicolumn{2}{|c|}{$P_{\text {III }}$} \\
\hline & $N_{I}$ & $N_{\text {II }}$ & & $N_{\text {II }}$ & $N_{\text {III }}$ \\
\hline $\begin{array}{l}\text { Poids }(\mathrm{g}) \ldots \ldots \ldots \\
\text { Taille }(\mathrm{cm}) \ldots \ldots \ldots\end{array}$ & $\begin{array}{c}2522 \pm 268 \\
46 \pm 2,4\end{array}$ & $\begin{array}{c}3166 \pm 228 \\
49,2 \pm 1,6\end{array}$ & $\begin{array}{c}3309 \pm 317 \\
49,7 \pm 1,9\end{array}$ & $\begin{array}{c}3431 \pm 286 \\
50,2 \pm 1,4\end{array}$ & $\begin{array}{c}4163 \pm 356 \\
52,1 \pm 1,4\end{array}$ \\
\hline
\end{tabular}


3 enfants se sont en fait retrouvés au-dessous des normes standards, vu le faible effectif, nous n'en avons pas constitué un sous-groupe. Le tableau 2 donne le poids ef la taille des mères. La moyenne est significativement différente en ce qui concerne les groupes $P_{I}, P_{I I}$ et $P_{I I I}$, autant pour la taille que pour le poids.

\section{TABLEAU 2}

Poids ef taille des mères

\begin{tabular}{|c|c|c|c|c|c|}
\hline & \multicolumn{5}{|c|}{ Groupes maternels } \\
\hline & \multicolumn{2}{|c|}{$\mathrm{PI}_{\mathrm{I}}$} & \multirow{2}{*}{$\frac{P_{I I}}{N_{I I}}$} & \multicolumn{2}{|c|}{$P_{I I I}$} \\
\hline & $\begin{array}{c}N_{I} \\
n=18\end{array}$ & $\begin{array}{c}\mathrm{N}_{\mathrm{II}} \\
\mathrm{n}=34\end{array}$ & & $\begin{array}{c}N_{\text {II }} \\
n=47\end{array}$ & $\begin{array}{c}N_{\text {III }} \\
n=19\end{array}$ \\
\hline $\begin{array}{l}\text { Poids }(\mathrm{kg}) \ldots \ldots \ldots \ldots \\
\text { Taille }(\mathrm{cm}) \ldots \ldots \ldots \ldots \\
\text { PP } 30 \mathrm{~s}^{*} \ldots \ldots \ldots \ldots \\
\text { PP totale } \ldots * \ldots \ldots \ldots\end{array}$ & $\begin{array}{r}49,8 \pm 4,6 \\
160,1 \pm 6,1 \\
7,2 \pm 3,4 \\
8,6 \pm 4,2\end{array}$ & $\begin{array}{r}48,2 \pm 4,8 \\
159,8 \pm 6,4 \\
8,0 \pm 2,1 \\
11,4 \pm 3,8\end{array}$ & $\begin{array}{r}56,0 \pm 7,1 \\
162,3 \pm 7,7 \\
9,3 \pm 2,2 \\
13,6 \pm 2,9\end{array}$ & $\begin{array}{r}59,4 \pm 11,7 \\
162,0 \pm 5,3 \\
13,7 \pm 3,6 \\
17,4 \pm 4,8\end{array}$ & $\begin{array}{r}61,7 \pm 6,8 \\
163,5 \pm 4,6 \\
13,0 \pm 2,9 \\
19,9 \pm 3,9\end{array}$ \\
\hline
\end{tabular}

* Prise de poids à 30 semaines (PP).

** Prise de poids au cours de la gestation (PP totale).

Dans le groupe $P_{I}$, le poids ef la taille des deux groupes de mères $\left(P_{I} N_{I}\right.$ ef $\left.P_{I} N_{I I}\right)$ sont identiques ef cela par définition, puisqu'il s'agissait d'un critère d'inclusion. La prise de poids à 30 semaines est légèrement supérieure pour le groupe $P_{I} N_{I r}$, mais

TABLEAU 3

Moyenne des rations caloriques maternelles

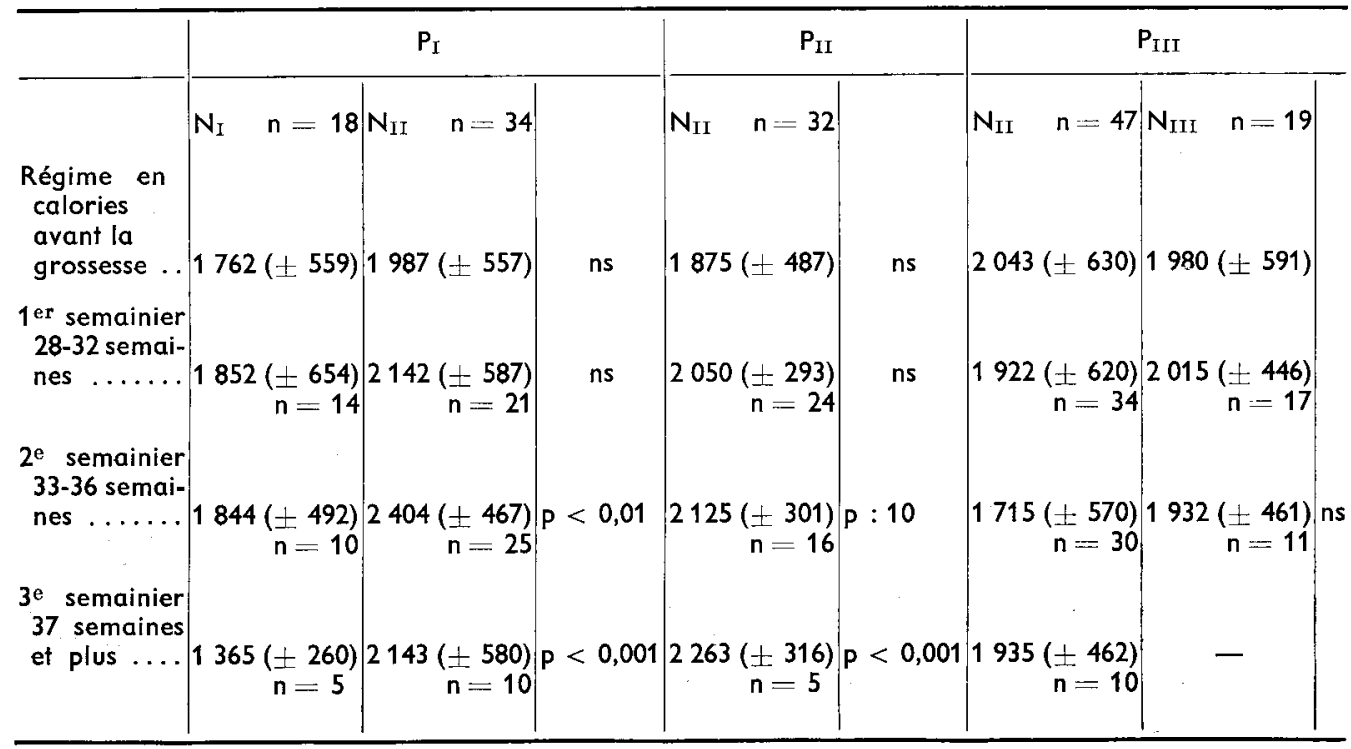


l'écart du gain pondéral au cours du troisième trimestre s'accroît de façon notable. Autrement dit, alors que la corpulence de ces femmes était à peu près identique au départ, il s'est passé quelque chose au cours du troisième trimestre de la gestation qui aboutit dans le groupe $P_{I} N_{I}$ à $8,6 \mathrm{~kg}$ de gain pondéral total et dans le groupe $P_{I} N_{I I}$ à $11,4 \mathrm{~kg}$. Dans le groupe $P_{\mathrm{II}}$, les résultats concordent avec les données de la littérature qui veulent qu'au cours de la grossesse normale le gain pondéral satisfaisant se situe entre 9 et $13 \mathrm{~kg}$ (Maternal Nutrition, 1970). Le groupe $P_{\text {III }}$ conduit aux mêmes constatations que le groupe $P_{I}$, mais bien sûr, avec une image en miroir : taille et poids des mères au départ identiques (par définition), prise de poids à 30 semaines identique mais prise de poids totale plus importante dans le groupe $P_{I I I} N_{I I I}(14,9 \mathrm{~kg})$ par rapport au groupe $P_{I I I} N_{I I}(17,4 \mathrm{~kg})$.

Le tableau 3 donne l'analyse du régime alimentaire des groupes ainsi définis. Pour les femmes du groupe témoin $P_{I I}$ on constate une augmentation de la ration calorique au cours de la gestation, ce qui correspond tout à fait aux notions classiques (Maternal Nutrition, 1970).

Pour celles du groupe $P_{I}$, on note une différence de départ, les femmes du sousgroupe $P_{I} N_{I}$ mangent près de 200 calories de moins que celles du sous-groupe $P_{I} N_{I I}$. Lorsque l'on suit l'évolution de la ration calorique du sous-groupe $P_{I} N_{I}$, on perçoit une très faible augmentation de celle-ci, au cours des 2 premiers semainiers, puis on enregistre une chute au $3^{\mathrm{e}}$ semainier (chaque semainier étant effectué à 1 mois d'inter-

TABLEAU 4

Composition du régime alimentaire dans le groupe I

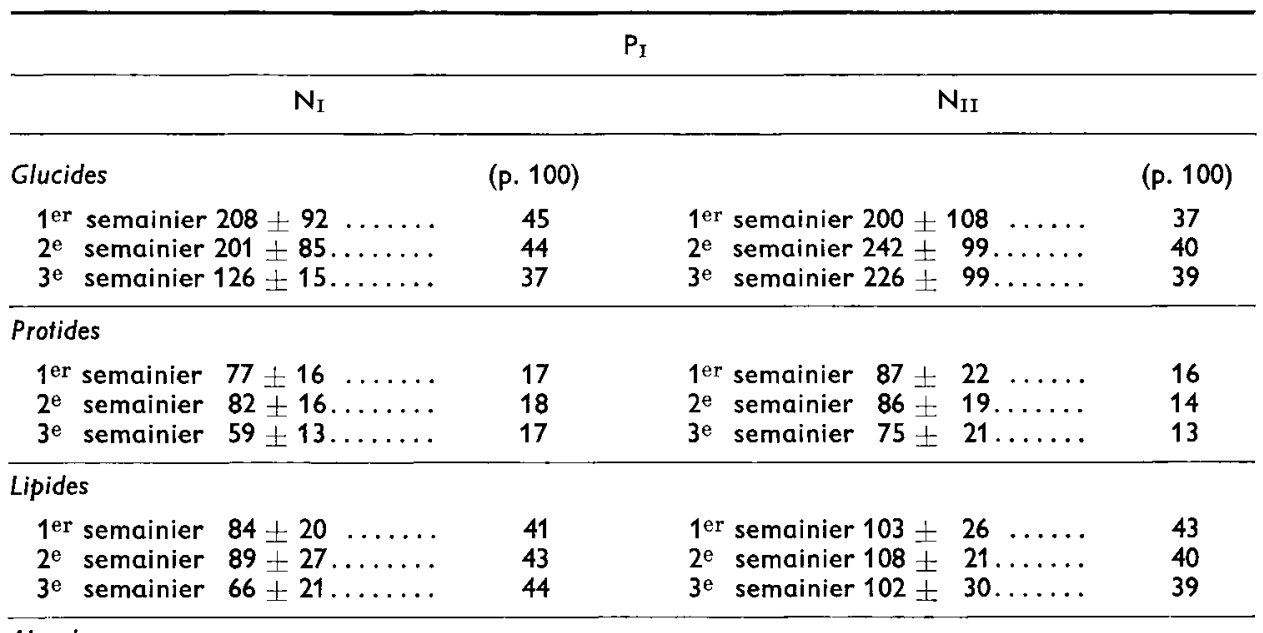

Alcool

(gr)

$\begin{array}{ll}0,1 & 0,4 \\ 0,2 & 0,6 \\ 0,0 & 0,4\end{array}$

Tabac 
valle). Il existe, dans le groupe $P_{I} N_{I I}$, à l'inverse, une augmentation au cours des 2 premiers semainiers et même au $3^{\mathrm{e}}$ semainier. Au vu de ces chiffres, il nous semble légitime de formuler qu'en dehors des autres causes de l'hypotrophie fotale (malformation fotale, malformation utérine, hypertension maternelle, etc...), une mère de faible corpulence dont la ration calorique avant la grossesse est déjà faible et qui, au cours de cette gestation ne va pas augmenter cette ration calorique ou va la diminuer, a le plus de chances d'obtenir un enfant de faible poids à la naissance.

Inversement, une mère de faible corpulence mais dont le régime alimentaire va s'accroître au cours de la gestation, a moins de chances d'obtenir un enfant de faible poids à la naissance. Ceci permet peut-être de conjuguer la part génétique qui revient à cette régulation du poids de naissance et la part qui revient à l'environnement maternel au cours de ce moment critique qu'est la croissance du foetus au cours du troisième trimestre de la gestation.

Le tableau 4 analyse la composition des régimes alimentaires. Il existe une reiative stabilité du pourcentage des protides dans les deux groupes de même que des lipides. Par contre la consommation de glucides chute progressivement dans le sous-groupe $P_{I} N_{I}$, alors qu'elle se maintient stable dans le sous-groupe $P_{I} N_{I I}$. Autrement dit, la ration alimentaire des femmes de petite corpulence et qui donneront naissance à des enfants de petit poids a globalement diminué au cours de la gestation ef cette diminution a été supportée essentiellement par les glucides. Cette restriction glucidique confirme une étude antérieure qui démontrait que les femmes porteuses d'enfants hypotrophiques réduisaient spontanément la ration calorique aux dépens des glucides afin de prévenir une difformité résiduelle due à la grossesse (Frydman et al., 1977). ii est à noter que les mères du groupe $P_{I} N_{I}$ semblent fumer plus que les autres. Par contre, la consommation d'alcool est à peu près identique.

La figure 1 visualise l'évolution du régime alimentaire du groupe $P_{I}$, en fonction de l'état du nouveau-né à la naissance. Lorsque l'on fait la moyenne des semainiers, en partant d'un point fictif qui est le point avant la grossesse, on constate une augmentation moyenne des calories plus importante dans le groupe $P_{I} N_{I I}$ que dans le groupe $P_{I} N_{I}$ puisque celui-ci est, même à 37 semaines en dessous du niveau antérieur à la grossesse.

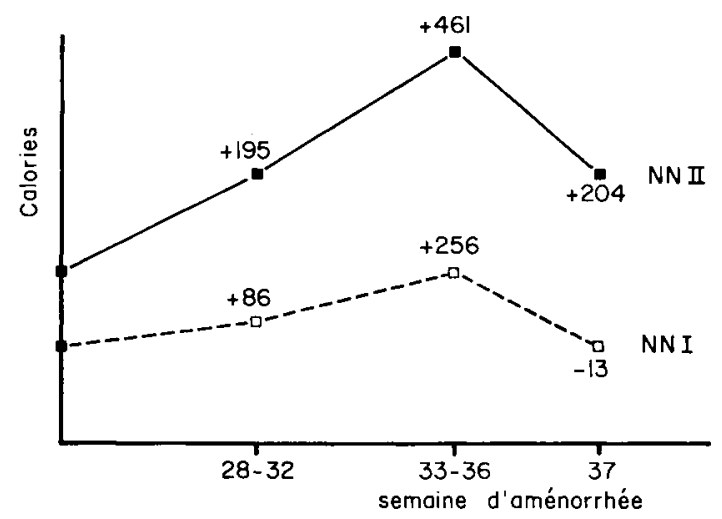

FIG. 1. - Variations de régime alimentaire du groube $P_{I}$. 
Si nous analysons le groupe $P_{I I I}$, les moyennes des rations caloriques ne sont pas très différentes. Cependant, il est à noter un abaissement progressif de ces moyennes du premier au deuxième semainier dans le groupe $P_{I I I} N_{I I}$ avec, paradoxalement, une remontée au cours du troisième semainier. On constate dans le groupe $P_{\text {III }} N_{\text {III }}$ une stabilité de la ration calorique. Cependant, la figure 2 montre que seulement 29 p. 100 des femmes du groupe $P_{\text {III }} N_{\text {III }}$ ont diminué leur régime alimentaire contre 70 p. 100 dans le groupe $P_{\text {III }} N_{\text {III }}$. Cette différence de pourcentage se retrouve lors de l'analyse du deuxième semainier. Autrement dit, les femmes obèses font relativement attention puisqu'elles augmentent peu leur ration calorique pendant la gestation. Cependant, le comportement individuel de ces femmes montre que celles qui ont réduit leur ration calorique au cours du $2^{\mathrm{e}}$ trimestre de la gestation ont dans l'ensemble, eut plus fréquemment des nouveau-nés de poids normal comparativement à celles qui n'ont pas - ou peu - réduit leur ration calorique.

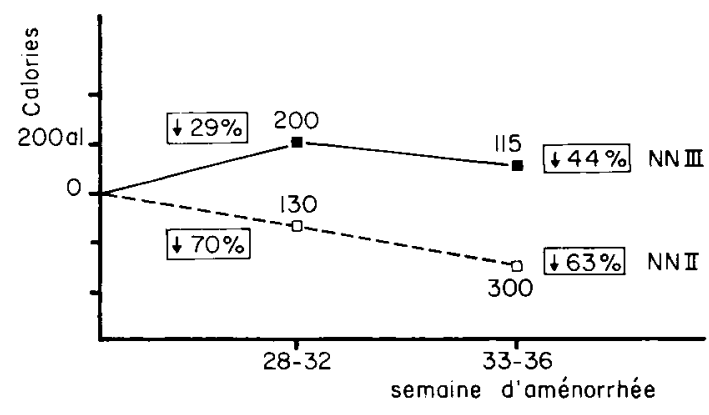

FIG. 2. - Variations de régime alimentaire du groupe $\mathrm{P}_{\mathrm{III}}$.

\section{Conclusion.}

Ces données qui représentent une des rares études prospectives faites sur ce domaine difficile de l'alimentation au cours de la grossesse montrent qu'en dehors de toute cause pathologique pouvant altérer la croissance fœetale, celle-ci est fonction de la corpulence maternelle. Mais une donnée nouvelle mérite d'être soulignée.

A l'intérieur d'un groupe de mères de corpulence donnée, il existe une modulation de la croissance foetale fonction des apports caloriques au cours de la gestation et tout particulièrement au cours du troisième trimestre de celle-ci qui représente, on le sait, la phase de croissance rapide du fœtus. Plus qu'un chiffre de calories globales à donner, il importe de s'attacher à l'augmentation ou à la diminution de la ration calorique au cours de la grossesse pour chaque femme. Il s'agit ici d'un comportement alimentaire individuel, car il existe de grandes variations du taux de calories ingérées par individu.

Une femme de faible corpulence si elle a un régime alimentaire satisfaisant et donc un gain pondéral également satisfaisant, a moins de chance de donner naissance à un enfant de faible poids.

De même, une femme chez qui on peut prévoir un gros enfant peut, par le simple contrôle de sa ration alimentaire, éviter un surpoids fœetal. Ceci témoigne de l'intérêt 
de l'introduction de consultations de diététique au sein des consultations prénatales, afin de préciser le statut alimentaire des femmes gestantes et d'y apporter une éventuelle correction.

$4^{\text {e }}$ Réunion du groupe Développement I.N.R.A., Montpellier, 17-18 mai 1978.

\section{Références}

BERGNER L., SUSSER M., 1970. Low birth weight and prenatal nutrition and interpretation review. Pediatrics, 46, 946-965.

EASTMAN N. J., JACKSON E., 1968. Weight relationship in pregnancy. Obsfef. Gynec., 23, 1003-1025.

FITZHARDINGE P. M., STEVEN E. M., 1972. The small for date infant. II. - Neurochirurgical and intellectual sequalae. Pediatrics, 50, 50-57.

FRYDMAN R., HAJERI H., PAPIERNIK E., 1977. Retard de croissance intra-utérin et nutrition prénatale. J. Gynecol. Biol. Reprod., 6, 1123-1132.

HYTTEN F. E., LEITCH I., 1971. Physiology of human pregnancy. 2nd Ed. Blackwell Sci. Publ., Oxford.

LECHTIG A., DELGADO H., LASKY R., 1975. Nutrition and fetal growth in developping societies. Am. J. Dis. Child., 129, 434-437.

LEROY B., LEFORT F., 1971. A propos du poids et de la taille des nouveau-nés à la naissance. Rev. franç. Gynécol., 66, 391-396.

Maternal nutrition and the course of pregnancy, 1970. Nat. Acad. Sci., Washington D. C., Govt. Prtg. Off.

NAEYE R. L., BLANC W., PAUL C., 1973. Effects of maternal nutrition on the human foetus. Pediatrics, 52, 494-503.

PEQUINOT G., CUBEAU J., 1973. Enquêtes méthodologiques comparant chez les mêmes sujets la consommation alimentaire appréciée par interrogatoire, à la consommation mesurée par pesée. Rev. épidém. Méd. soc. Santé publ., 21, 585-608.

SACK R. A., 1969. The large infant. Am. J. Obstetr. Gynecol., 104, 195-204.

SINGER J. E., WESTPHAL M., NISWANDER K., 1968. Relationship of weight gain during pregnancy to birth weight and infant growth and development in the first year of life. Obstet. Gynecol., 31, 417-423.

THOMSON A. M., HYTTEN F. E., 1973. Nutrition in pregnancy. Food nutrition and health. WId Rev. Nutr. Diet., 16, 22-45. M. Recheigl, Washington ; Karger, Basel.

WYNN M., WYNN A., 1975. Nutrition counselling in the prevention of low birth weight. Foundation for education and research in child-bearning, London. 